\title{
Prognostic factors and outcomes in anaplastic gliomas: An institutional experience
}

\author{
Deepthi Valiyaveettil, Monica Malik, Deepa Joseph, Syed Fayaz Ahmed, Syed Akram Kothwal
}

\begin{abstract}
Background: There is lack of clear evidence and treatment guidelines for anaplastic gliomas (AGs) with very few studies focusing exclusively on these patients. The aim of the study was to analyze the clinical profile and survival in these patients. Materials and Methods: Patients of AGs treated with radiation and concurrent \pm adjuvant chemotherapy from January 2010 to December 2015 were analyzed. Statistical analysis was done using SPSS version 20 software. Results: A total of 100 patients were included in the study. The median age was 35 years (range 6-68 years). Eighty-four patients had follow-up details and were included for survival analysis. The 5 -year overall survival (OS) was $58 \%$. Age, presentation with seizures, and focal neurological deficit were not found to significantly influence survival. The 5-year survival for oligodendroglioma and astrocytoma was $69 \%$ and $52 \%$, respectively. Patients with Karnofsky Performance Score (KPS) of $\geq 70$ had a significantly better 5 -year OS (65\%) as compared to those with KPS $<70(33 \%)(P=0.000)$. The use of adjuvant temozolomide (TMZ) showed longer 5 -year OS of $67.7 \%$ compared to $36 \%$ in patients who did not receive adjuvant chemotherapy $(P=0.018)$. Patients receiving both concurrent and adjuvant TMZ showed longer 5 -year OS $(68.5 \%$ vs. $40 \%, P=0.010)$. Twenty-two patients had recurrence with average time to recurrence being 37 months. Fourteen patients underwent salvage surgery and two patients received reirradiation. Conclusions: OS significantly correlated with KPS and receipt of concurrent and adjuvant chemotherapy with TMZ. Therefore, adjuvant radiation with concurrent and adjuvant TMZ should be the standard of care for AGs.
\end{abstract}

Key words: Anaplastic gliomas, chemoradiation, survival, temozolomide

\section{Introduction}

Gliomas account for $80 \%$ of primary malignant brain tumors. ${ }^{[1]}$ Anaplastic gliomas (AGs) constitute $6.1 \%$ of all primary central nervous system gliomas. AGs are typically treated with maximal safe resection, followed by external beam radiation therapy (RT). This approach is supported by observational data that suggest that the survival of patients with AGs is longer after complete or near-total resection (compared to biopsy alone). ${ }^{[2]}$ Randomized controlled trials of patients with high-grade gliomas suggest that RT is associated with longer survival. ${ }^{[3,4]}$ Currently, there limited evidence for AG despite the widespread use of chemotherapy to treat these cancers. No consensus has been derived from a previously conducted survey of recommendations for treatment of newly diagnosed AG. ${ }^{[5]}$

The aim of the study was to analyze the clinical profile, and treatment outcomes of patients with AGs treated with concurrent chemoradiation (CRT) at our institute.

\section{Materials and Methods}

Medical records of patients with histopathological diagnosis of AGs treated with adjuvant radiotherapy with or without concurrent and adjuvant chemotherapy between 2010 and 2015 were reviewed. Clinical data such as age, performance status, and extent of resection were collected. The extent of surgery was identified as gross total resection, subtotal resection or biopsy by operative and clinical notes and when available by postoperative imaging. Patient-, tumor-, and treatment-related variables were recorded and used to assign patients to an RT Oncology Group recursive partitioning analysis (RTOG RPA) classification. All patients received external beam RT to a total dose of 60 Gy, 2 Gy per fraction for 30 fractions. Most patients were treated using intensity-modulated radiotherapy technique. All patients completed the planned course of radiation without any significant interruptions. Concurrent

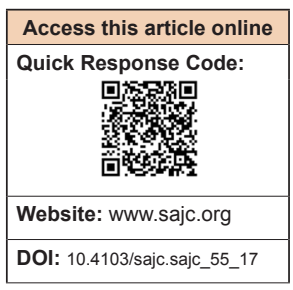

Department of Radiation Oncology, Nizam's Institute of Medical Sciences, Hyderabad, Telangana, India Correspondence to: Dr. Monica Malik, E-mail: dr_monical I@yahoo.com chemotherapy regimen prescribed was temozolomide (TMZ) to a dose of $75 \mathrm{mg} / \mathrm{m}^{2} /$ day PO for the entire course of radiation. Following CRT, all patients were advised adjuvant TMZ (150-200 mg/m²) 5 days a week every 28 days for $6-12$ cycles. Data that were unavailable in the medical records due to follow-up loss were obtained via a telephone interview with the patient or, if the patient was deceased, with his or her relatives with their permission. Overall survival (OS) was defined as the time from the date of diagnosis to the date of death or last contact.

\section{Statistical analysis}

Kaplan-Meier estimates were used to analyze OS estimates with the comparison of rates among the groups performed using the two-tailed log-rank test. A two-sided $P<0.05$ was considered statistically significant. The analysis was done using SPSS version 20 software (IBM corporation).

\section{Results}

A total of 100 patients were included in this analysis. The median age was 35 years ranging from 6 to 68 years. Nine patients were $<18$ years of age, 48 patients were $<35$ years of age and 52 patients were 35 years or above. About $61 \%$ were males and $39 \%$ were females with male:female ratio of 1.6:1. Most common presentation was with headache $(65 \%)$, seizures (44\%), vomiting (21\%), or hemiparesis (19\%). Average duration of symptoms was 8 months. Twenty-nine patients had focal neurological deficit at the time of presentation. Most common location of tumor was the frontal lobe followed by temporal lobe. Fifty-seven patients had tumors with contrast enhancement. Majority of the patients belonged to RTOG RPA class I. Patient characteristics are tabulated in Table 1.

Based on the surgical notes, gross total resection was done in 22 patients, near-total resection in 29 patients, subtotal resection in

This is an open access article distributed under the terms of the Creative Commons Attribution-NonCommercial-ShareAlike 3.0 License, which allows others to remix tweak, and build upon the work non-commercially, as long as the author is credited and the new creations are licensed under the identical terms.

For reprints contact: reprints@ @medknow.com
How to cite this article: Valiyaveettil D, Malik M, Joseph D, Ahmed SF, Kothwal SA. Prognostic factors and outcomes in anaplastic gliomas: An institutional experienceLetter to the Editor. South Asian J Cancer 2018;7:1-4. 
Table 1: Patient characteristics

\begin{tabular}{|c|c|}
\hline Characteristics & Number of patients \\
\hline \multicolumn{2}{|l|}{ Age } \\
\hline Median & 35 years \\
\hline Range & $6-68$ years \\
\hline$<35$ years & 48 \\
\hline$\geq 35$ years & 52 \\
\hline \multicolumn{2}{|l|}{ Sex } \\
\hline Male & 61 \\
\hline Female & 39 \\
\hline Ratio & $1.56: 1$ \\
\hline \multicolumn{2}{|l|}{ Clinical presentation } \\
\hline Headache & 65 \\
\hline Seizures & 44 \\
\hline Vomiting & 21 \\
\hline One-sided weakness & 19 \\
\hline Blurring vision & 10 \\
\hline \multicolumn{2}{|c|}{ Focal neurological deficit } \\
\hline Yes & 29 \\
\hline No & 71 \\
\hline \multicolumn{2}{|c|}{ Location on MRI/CT scan } \\
\hline Frontal lobe & 54 \\
\hline Temporal lobe & 20 \\
\hline Parietal lobe & 15 \\
\hline Thalamus & 6 \\
\hline Cerebellum & 5 \\
\hline \multicolumn{2}{|l|}{ RTOG RPA class } \\
\hline I & 79 \\
\hline II & 2 \\
\hline III & 5 \\
\hline IV & 10 \\
\hline $\mathrm{V}$ & 4 \\
\hline
\end{tabular}

RTOG=Radiation therapy oncology group, RPA=Recursive partitioning analysis, MRI=Magnetic resonance imaging, $\mathrm{CT}=$ Computed tomography

38 patients and biopsy/decompression in 11 patients. Most of the histopathological diagnosis was morphological. Sixty-three patients had AA histology, three cases were reported as oligoastrocytoma and 37 patients had AO histology. Fourteen patients were tested for IDH mutation of whom nine were positive. Three out of six patients tested for $1 \mathrm{p} 19 \mathrm{q}$ codeletion were positive.

Out of 100 patients, 82 received concurrent chemotherapy with TMZ and 62 patients received concurrent and adjuvant TMZ. Median number of adjuvant chemotherapy cycles was five. Treatment details are tabulated in Table 2.

Out of 100 patients, follow-up details were available for 84 and these patients were included in the survival analysis. Median OS was not yet reached at the time of analysis. The 5-year OS was $58 \%$ [Figure 1a]. The Kaplan-Meier survival estimates for other cofactors are tabulated in Table 3.

Younger age, female sex, presentation with seizures, and no focal neurological deficit showed a trend to longer survival but were not statistically significant. Surgical extent defined by operating surgeon did not correlate with survival. Probable explanation for this observation could be that the extent of resection might be overestimated at the time of surgery and immediate postoperative magnetic resonance imaging (MRI) could not be done in all cases. Patients with postoperative residual tumor on imaging had a shorter survival.

Patients with AO histology had 5-year OS of $65.7 \%$ compared to $51.9 \%$ for AA (log-rank $P=0.27)$. The use of concurrent
Table 2: Treatment details

\begin{tabular}{lc}
\hline Other characteristics & Number of patients \\
\hline Surgery & 22 \\
GTE & 29 \\
NTE & 38 \\
STE & 11 \\
Biopsy/decompression & \\
Histopathology & 37 \\
AO & 60 \\
AA & 3 \\
OA & \\
Postoperative residual & 70 \\
Yes & 30 \\
No & \\
RT technique & 11 \\
2D & 22 \\
3DCRT & 67 \\
IMRT & \\
Chemotherapy & 82 \\
Concurrent & 62 \\
Concurrent + adjuvant & 5.2 \\
Average adjuvant cycles & \\
Residual on follow-up scan & 34 \\
Yes & 66 \\
No & \\
\hline RT=Radiation therapy, IMRT=Intensity modulated radiation therapy, \\
DCRT=Dimensional conformal radiation therapy, 2D=Two-dimensional, \\
AA=Anaplastic astrocytoma, AO=Anaplastic oligodendroglioma, \\
OA=Oligoastrocytoma, GTE=Gross total excision, STE= Sub total excision, \\
NTE=Near total excision
\end{tabular}

$\mathrm{NTE}=$ Near total excision

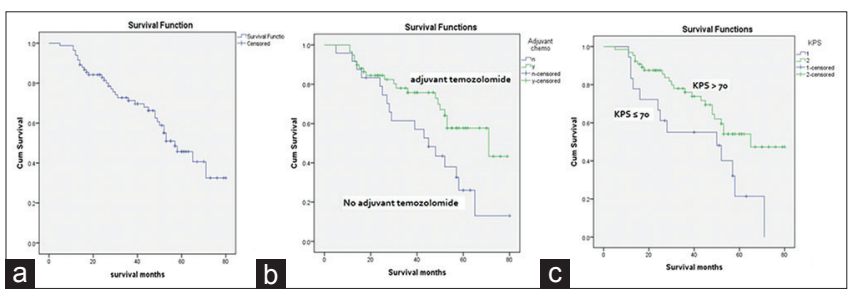

Figure 1: (a) Overall survival by Kaplan-Meier method. (b) Kaplan-Meier survival analysis by chemotherapy. (c) Kaplan-Meier survival analysis by Karnofsky Performance score

chemotherapy with TMZ showed a longer 5-year OS compared to only RT $(59.1 \%$ vs. $53.8 \%$, log-rank $P=0.63)$. The use of adjuvant chemotherapy with TMZ showed longer 5-year OS of $67.7 \%$ compared to $36 \%$ in patients with no adjuvant chemotherapy (log-rank $P=0.018$ ). Patients receiving both concurrent and adjuvant TMZ also showed longer 5-year OS $(68.5 \%$ vs. $40 \%, P=0.010)$ [Figure $1 \mathrm{~b}$ ]. There were no grade 3 or higher toxicities noted with this regimen.

Patients with preradiotherapy Karnofsky Performance Score (KPS) $\leq 70$ had 5 -year OS of $33.3 \%$ and those with KPS $>70$ had 5 -year OS of $65.1 \%$ (log-rank $P=0.000$ ) [Figure 1c]. A follow-up MRI done at 6-month post-RT showed residual tumor in 56 patients. Patients with no residual had 5-year OS of $66.7 \%$ and patients having residual on follow-up scan had 5-year OS of $54.3 \%$ (log-rank $P=0.19$ ). Twenty-three patients had documented recurrence during with average time to recurrence being 37 months. Fourteen patients underwent salvage surgery and 3 patients received reirradiation. The average survival after recurrence was 12.7 months.

South Asian Journal of Cancer $\bullet$ Volume 7 Issue 1 • January-March 2018 
Table 3: Kaplan-Meier survival estimates

\begin{tabular}{|c|c|c|}
\hline Cofactor & 5 -year OS in \% & Log-rank $P$ \\
\hline \multicolumn{3}{|c|}{ Age (years) } \\
\hline$<35$ & 59.5 & 0.83 \\
\hline$\geq 35$ & 57.1 & \\
\hline \multicolumn{3}{|l|}{ Sex } \\
\hline Male & 53.8 & 0.38 \\
\hline Female & 65.6 & \\
\hline \multicolumn{3}{|c|}{ Seizures at presentation } \\
\hline Yes & 58.9 & 0.76 \\
\hline No & 57 & \\
\hline \multicolumn{3}{|l|}{ FND } \\
\hline Yes & 46.1 & 0.27 \\
\hline No & 63.7 & \\
\hline \multicolumn{3}{|l|}{ KPS } \\
\hline$\leq 70$ & 33.3 & 0.01 \\
\hline$>70$ & 65 & \\
\hline \multicolumn{3}{|c|}{ Enhancement on contrast } \\
\hline Yes & 52 & 0.15 \\
\hline No & 66 & \\
\hline \multicolumn{3}{|c|}{ Histopathology } \\
\hline AA & 51.9 & 0.27 \\
\hline $\mathrm{AO}$ & 68.7 & \\
\hline \multicolumn{3}{|c|}{ Concurrent TMZ } \\
\hline Yes & 59.1 & 0.63 \\
\hline No & 53.8 & \\
\hline \multicolumn{3}{|c|}{ Adjuvant TMZ } \\
\hline Yes & 67.7 & 0.01 \\
\hline No & 36.6 & \\
\hline \multicolumn{3}{|c|}{ Both adjuvant and concurrent } \\
\hline Yes & 68.5 & 0.01 \\
\hline No & 40 & \\
\hline \multicolumn{3}{|l|}{ Residual } \\
\hline Yes & 54.3 & 0.19 \\
\hline No & 66.6 & \\
\hline
\end{tabular}

\section{Discussion}

Only sparse evidence exist that address the management of AG. Until recently most trials combined both grades III and IV gliomas as malignant glioma. Therefore, some of the conclusions are based on extrapolation from high-grade glioma in general and may not be entirely true for AGs which are prognostically different from grade IV gliomas. A review of published data on high-grade gliomas in India showed that most studies were focused on mixed population of AG and glioblastoma and there was no study which reported data exclusively on AG. ${ }^{[6]}$ The reported 5-year survival rates of $A G$ patients have been $<40 \%$ despite multimodality treatments such as surgery and adjuvant therapy. ${ }^{[7]}$

Age, neurological deficit, extent of surgery, and KPS influence prognosis in adult patients. ${ }^{[8-11]}$ Younger age and no focal neurological deficit showed a longer survival in our study.

Oligodendrogliomas are known to be chemosensitive and respond to treatment although the best treatment for these tumors is controversial. ${ }^{[12]}$ Codeletion of $1 \mathrm{p} 19 \mathrm{q}$ among AO patients has been validated as an independent and significant predictor of improved survival. Estimates of median OS vary widely and range from 4.45 to 65.55 months for AA and 14.4 to 184.41 months for AO tumors, with some AO South Asian Journal of Cancer $\bullet$ Volume 7 Issue 1 January-March 2018 patients $(1 \mathrm{p} / 19 \mathrm{q}$ codeleted tumors) demonstrating median survivals of more than 10 years. ${ }^{[13,14]}$

Better prognosis of oligodendroglial tumors over astrocytic tumors is also supported by previous studies. ${ }^{[15]}$ Moreover, the diffuse nature of AA usually precludes a complete resection and may be responsible for inferior outcomes. Longer survival of oligodendroglial tumors over anaplastic astrocytomas was observed in our study, although this difference did not reach statistical significance. The nonavailability of details on possible allelic losses on chromosomes arms $1 \mathrm{p}$ and $19 \mathrm{q}$ and IDH mutation status in our patient population prevents us from fully assessing this observed survival disparity after RT.

It is becoming increasingly common to incorporate $\mathrm{TMZ}$ with radiation for patients with AA through extrapolation of results from the landmark Stupp trial. ${ }^{[16]}$ In the landmark, RTOG 9402 and EORTC26981 studies chemosensitivity to PCVProcarbazine, Lomustine, Vincristine (PCV) was not observed in patients with noncodeleted $\mathrm{AO} \cdot{ }^{[13,17]}$

TMZ has largely replaced PCV in the treatment of gliomas. However, controversy remains whether TMZ efficacy is equivalent to that of $\mathrm{PCV} .^{[7]} \mathrm{TMZ}$ and RT have been widely used in the treatment of glioblastoma and successful outcomes have been reported in retrospective series of AGs. Because AGs often transform to glioblastoma, some have speculated that a similar upfront treatment approach is warranted in AGs. Moreover, studies have demonstrated favorable results when TMZ is used for recurrent AGs. However, the effect of using TMZ during and after RT for AGs has not been well studied. [18-20]

A retrospective study on 165 AA patients examining concurrent TMZ with adjuvant RT, found that concurrent TMZ was significantly associated with improved 5-year OS compared to those receiving RT alone $(46.2 \%$ vs. $29.3 \%, P=0.02){ }^{[21]}$ In our study, concurrent TMZ showed improved survival, but this was not statistically significant.

Two ongoing trials CATNON and CODEL are expected to provide a consensus to many of the controversies. The CATNON trial included patients with $A G$ without $1 \mathrm{p} / 19 \mathrm{q}$ codeletion. ${ }^{[22]}$ Interim analysis ${ }^{[23]}$ suggests 12 cycles of adjuvant TMZ improved OS. In this study, patients who received adjuvant TMZ had longer 5-year OS compared to patients who did not receive adjuvant TMZ $(67.7 \%$ vs. $36 \%$, log-rank $P=0.01)$.

The CODEL trial is an ongoing trial in codeleted patients and is examining RT followed by PCV versus TMZ concurrent with RT followed by adjuvant TMZ versus TMZ alone. ${ }^{[22]}$

Limitations of our study include lack of molecular information such as IDH mutation status, MGMT methylation, 1p19q codeletion, ATRX, and C-GIMP which are established prognostic markers.

\section{Conclusions}

Five-year OS in our patients was 58\% which is higher than historically reported data. Age $>35$ years, no neurological deficit at presentation, oligodendroglioma histology, and KPS $>70$ were associated with better OS. Adjuvant radiotherapy with concurrent and adjuvant TMZ showed significant improvement in OS. Results from the ongoing randomized trials are likely to provide better insights into the optimal combinations of adjuvant therapies in these tumors. 
Financial support and sponsorship

Nil.

Conflicts of interest

There are no conflicts of interest.

\section{References}

1. Ricard D, Idbaih A, Ducray F, Lahutte M, Hoang-Xuan K, Delattre JY, et al. Primary brain tumours in adults. Lancet 2012;379:1984-96.

2. Laws ER, Parney IF, Huang W, Anderson F, Morris AM, Asher A, et al. Survival following surgery and prognostic factors for recently diagnosed malignant glioma: Data from the Glioma Outcomes Project. J Neurosurg 2003;99:467-73.

3. Walker MD, Alexander E Jr., Hunt WE, MacCarty CS, Mahaley MS Jr., Mealey J Jr., et al. Evaluation of BCNU and/or radiotherapy in the treatment of anaplastic gliomas. A cooperative clinical trial. J Neurosurg 1978;49:333-43.

4. Kristiansen K, Hagen S, Kollevold T, Torvik A, Holme I, Nesbakken R, et al. Combined modality therapy of operated astrocytomas grade III and IV. Confirmation of the value of postoperative irradiation and lack of potentiation of bleomycin on survival time: A prospective multicenter trial of the Scandinavian Glioblastoma Study Group. Cancer 1981;47:649-52.

5. Abrey LE, Louis DN, Paleologos N, Lassman AB, Raizer JJ, Mason W, et al. Survey of treatment recommendations for anaplastic oligodendroglioma. Neuro Oncol 2007:9:314-8.

6. Dasgupta A, Gupta T, Jalali R. Indian data on central nervous tumors: A summary of published work. South Asian J Cancer 2016;5: 147-53.

7. DeAngelis LM. Anaplastic glioma: How to prognosticate outcome and choose a treatment strategy. [corrected]. J Clin Oncol 2009;27:5861-2.

8. Brandes AA, Nicolardi L, Tosoni A, Gardiman M, Iuzzolino P, Ghimenton C, et al. Survival following adjuvant PCV or temozolomide for anaplastic astrocytoma. Neuro Oncol 2006;8:253-60.

9. Stupp R, Reni M, Gatta G, Mazza E, Vecht C. Anaplastic astrocytoma in adults. Crit Rev Oncol Hematol 2007;63:72-80.

10. See SJ, Gilbert MR. Anaplastic astrocytoma: Diagnosis, prognosis, and management. Semin Oncol 2004;31:618-34.

11. Prados MD, Gutin PH, Phillips TL, Wara WM, Larson DA, Sneed PK, et al. Highly anaplastic astrocytoma: A review of 357 patients treated between 1977 and 1989. Int J Radiat Oncol Biol Phys 1992;23:3-8.

12. Gan HK, Rosenthal MA, Dowling A, Kalnins R, Algar E, Wong N, et al. A phase II trial of primary temozolomide in patients with grade III oligodendroglial brain tumors. Neuro Oncol 2010;12:500-7.
13. Cairncross G, Wang M, Shaw E, Jenkins R, Brachman D, Buckner J, et al. Phase III trial of chemoradiotherapy for anaplastic oligodendroglioma: Long-term results of RTOG 9402. J Clin Oncol 2013;31:337-43.

14. Parkinson JF, Afaghi V, Payne CA, Buckland ME, Brewer JM, Biggs MT, et al. The impact of molecular and clinical factors on patient outcome in oligodendroglioma from 20 years' experience at a single centre. J Clin Neurosci 2011;18:329-33.

15. Miller CR, Dunham CP, Scheithauer BW, Perry A. Significance of necrosis in grading of oligodendroglial neoplasms: A clinicopathologic and genetic study of newly diagnosed high-grade gliomas. J Clin Oncol 2006;24:5419-26.

16. Stupp R, Hegi ME, Mason WP, van den Bent MJ, Taphoorn MJ, Janzer RC, et al. Effects of radiotherapy with concomitant and adjuvant temozolomide versus radiotherapy alone on survival in glioblastoma in a randomised phase III study: 5-year analysis of the EORTC-NCIC trial. Lancet Oncol 2009; 10:459-66.

17. van den Bent MJ, Brandes AA, Taphoorn MJ, Kros JM, Kouwenhoven MC, Delattre JY, et al. Adjuvant procarbazine, lomustine, and vincristine chemotherapy in newly diagnosed anaplastic oligodendroglioma: Long-term follow-up of EORTC brain tumor group study 26951. J Clin Oncol 2013;31:344-50.

18. Yung WK, Prados MD, Yaya-Tur R, Rosenfeld SS, Brada M, Friedman HS, et al. Multicenter phase II trial of temozolomide in patients with anaplastic astrocytoma or anaplastic oligoastrocytoma at first relapse. Temodal Brain Tumor Group. J Clin Oncol 1999; 17:2762-71.

19. Smrdel U, Kovac V, Popovic M, Zwitter M. Glioblastoma patients in slovenia from 1997 to 2008. Radiol Oncol 2014;48:72-9.

20. Kim YH, Park CK, Cho WH, Kim IA, Moon S, Choe G, et al. Temozolomide during and after radiation therapy for WHO grade III gliomas: Preliminary report of a prospective multicenter study. J Neurooncol 2011; 103:503-12.

21. Kizilbash SH, Giannini C, Voss JS, Decker PA, Jenkins RB, Hardie J, et al. The impact of concurrent temozolomide with adjuvant radiation and IDH mutation status among patients with anaplastic astrocytoma. J Neurooncol 2014;120:85-93.

22. Weller M, van den Bent M, Hopkins K, Tonn JC, Stupp R, Falini A, et al. EANO guideline for the diagnosis and treatment of anaplastic gliomas and glioblastoma. Lancet Oncol 2014; 15:e395-403.

23. van den Bent MJ, Erridge S, Vogelbaum M, Nowak A, Sanson M, Brandes AA, et al. EH1.1 results of the interim analysis of the EORTC randomized phase III CATNON trial on concurrent and adjuvant temozolomide in anaplastic glioma without $1 p / 19 q$ co-deletion, an intergroup trial. Neuro Oncol 2016;18 Suppl 4:iv1. 